\title{
Impact of clinical trial participation on survival in patients with castration-resistant prostate cancer: a multi-center analysis
}

Kyo Chul Koo', Jong Soo Lee ${ }^{2}$, Jong Won Kim², Kyung Suk Han², Kwang Suk Lee', Do Kyung Kim', Yoon Soo Ha', Koon Ho Rha ${ }^{2}$, Sung Joon Hong ${ }^{2}$ and Byung Ha Chung ${ }^{*}$

\begin{abstract}
Background: Clinical trial (CT) participation may confer access to new, potentially active agents before their general availability. This study aimed to investigate the potential survival benefit of participation in investigational CTs of novel hormonal, chemotherapeutic, and radiopharmaceutical agents in patients with castration-resistant prostate cancer (CRPC).

Methods: This multi-center, retrospective analysis included 299 consecutive patients with newly diagnosed, nonmetastatic or metastatic CRPC between September 2009 and March 2017. Of these, 65 (21.7\%) patients participated in CTs pertaining to systemic treatment targeting CRPC and 234 (78.3\%) patients received pre-established, standard systemic treatment outside of a $C T$ setting. The survival advantage of $C T$ participation regarding cancer-specific survival (CSS) was investigated.

Results: An Eastern Cooperative Oncology Group performance status (ECOG PS) $\geq 2$ at CRPC diagnosis was found in a lower proportion CT participants than in non-participants (4.6\% vs. $14.9 \% ; p=0.033)$. During the median followup period of 16.0 months, CT participants exhibited significantly higher 2-year CSS survival rates (61.3\% vs. 42.4\%; $p$ $=0.003)$ than did non-participants. Multivariate analysis identified prostate-specific antigen and alkaline phosphatase levels at CRPC onset, Gleason score $\geq 8$, ECOG PS $\geq 2$, less number of docetaxel cycles administered, and non-participation in CTs as independent predictors for a lower risk of CSS.

Conclusions: Patients diagnosed with CRPC who participated in CTs exhibited longer CSS durations than nonparticipants who received pre-established, standard systemic therapy outside of a CT setting. Our findings imply that CT participation is associated with CSS, and that CT participation should be offered to patients with CRPC whenever indicated.
\end{abstract}

Keywords: Clinical trial, Prostatic neoplasms, Castration-resistant, Survival

\section{Background}

In line with advances in clinical research, the treatment of castration-resistant prostate cancer (CRPC) has evolved, with the development of novel hormonal, chemotherapeutic, radiopharmaceutical, and immunotherapeutic drugs [1]. Approval of these agents was based on the results of large, well-designed, randomized phase III

\footnotetext{
* Correspondence: chung646@yuhs.ac

'Department of Urology, Gangnam Severance Hospital, Yonsei University College of Medicine, 211 Eonju-ro, Gangnam-gu, Seoul 135-720, Republic of Korea

Full list of author information is available at the end of the article
}

clinical trials (CTs) that demonstrated improvement in overall survival [2-5]. However, there is still an unmet need to provide individualized therapeutic options, and the requirement for novel agents based on various pathways and targets continues to exist.

Participation in CTs may confer access to new, potentially active therapeutic agents before their general availability. Moreover, these investigational agents may be the best current treatment option for a subset of patients. Currently, there is ongoing research into and development of novel agents targeting CRPC, including androgen receptor inhibitors, cytochrome P450 17

(c) The Author(s). 2018 Open Access This article is distributed under the terms of the Creative Commons Attribution 4.0 International License (http://creativecommons.org/licenses/by/4.0/), which permits unrestricted use, distribution, and reproduction in any medium, provided you give appropriate credit to the original author(s) and the source, provide a link to the Creative Commons license, and indicate if changes were made. The Creative Commons Public Domain Dedication waiver (http://creativecommons.org/publicdomain/zero/1.0/) applies to the data made available in this article, unless otherwise stated. 
inhibitors, targeted agents, vaccines, and poly-ADTribose polymerase inhibitors [6-8]. A randomized CT is a crucial step in the development of new cancer treatments, and as with previously approved drugs, the efficacy of these novel agents will need to be confirmed by adequate statistical power through CTs before their application in clinical practice.

Physicians offer enrollment in CTs assuming that survival benefits may be obtained from participation. Participating in CTs may also provide patients with hope of an individualized survival benefit conferred by the use of potentially effective agents that would not have been received outside the trial setting. Indeed, several studies have shown entry into cancer CTs to be associated with increased survival rates $[9,10]$. On the other hand, concerns around the uncertainty associated with the experimental nature of CTs, the randomization process, unknown potential toxicity, and the time delay until proven standard therapy is available are documented barriers to enrollment [11-13].

With advances in understanding the mechanisms underlying castration-resistance and disease progression, CTs of investigational drugs targeted at CRPC will continue. Ideally, CTs should be offered as the best treatment option for patients based on evidence that participation may improve survival outcomes. Indeed, several investigations have reported a favorable overall trend with CT entry [14-16]. However, data on whether CTs targeted at CRPC may confer benefit in regard to survival are limited. The objective of this retrospective study was to determine the independent cancer-specific survival (CSS) advantage of participation in investigational CTs of hormonal, chemotherapeutic, and radiopharmaceutical agents targeted at CRPC.

\section{Methods}

\section{Study population}

A multicenter, retrospective analysis was performed using a prospectively collected database of 331 consecutive patients who were diagnosed with non-metastatic or metastatic CRPC between September 2009 and March 2017. Prostate cancer staging was based on the 7th American Joint Committee on Cancer TNM system, with the definition of bone metastasis based on either demonstrable metastatic deposits on imaging studies (bone scan, computed tomography, magnetic resonance imaging, or positron emission tomography) or by pathological confirmation. Patients were excluded from the analysis if they met any of the following criteria: incomplete clinical data $(n=14)$, lost to follow-up $(n=10)$, or unknown cause of death $(n=8)$.

Once diagnosed with CRPC, patients' eligibility for participation in available CTs pertaining to novel hormonal, chemotherapeutic, and radiopharmaceutical investigational agents was assessed. The eligibility criteria for 18 CTs are listed in Table 1. Patients who did not meet the eligibility criteria or who refused to participate in CTs received systemic treatment according to standard U.S. Food and Drug Administration-approved dose and schedule. The choice and sequencing of standard agents were based on physician discretion and patient preference. Each agent was continued until the occurrence of radiographic disease progression, intolerable side-effects, patient refusal, or death. Serum prostate-specific antigen (PSA) measurements were performed every 1 to 3 months, and computed tomography and bone scans were performed every 2 to 4 months. This study was approved by the Yonsei University Health System Institutional Review Board after a review of the study protocol (2017-0186-001).

\section{Data collection and definitions}

The patients' clinical and pathological characteristics at CRPC diagnosis were retrieved from the institutional electronic medical record database. The obtained data included patient age; body mass index; serum PSA level at CRPC diagnosis; Gleason score; AJCC stage; previous local treatments received; Charlson Comorbidity Index (CCI); Eastern Cooperative Oncology Group performance score (ECOG PS); the site of metastasis; duration of docetaxel, abiraterone, enzalutamide, cabazitaxel, and radium-223 dichloride administration; docetaxel to androgen receptor axis-targeted agent sequencing; and laboratory values including peripheral blood hemoglobin, albumin, and alkaline phosphatase levels, and white blood cell counts.

CRPC was defined and evaluated according to the criteria of the Prostate Cancer Clinical Trials Working Group 2 [17]. The CSS interval was defined as the interval from the date of initial CRPC diagnosis to the date of death from prostate cancer. Patient survival and causes of death were investigated based on the National Cancer Registry Database or institutional electronic medical records.

\section{Statistical analysis}

Clinicopathologic data were compared between CT participants and non-participants using descriptive statistics. Fisher's exact test and the chi-squared test were used to compare categorical variables. The Mann-Whitney U-test was used to compare continuous variables across dichotomous categories. Kaplan-Meier curves were used to estimate CSS according to CT participation, with $p$-values computed using the log-rank test.

Univariate and multivariate Cox proportional hazards regression analyses were used to adjust for potential confounders in predicting CSS. All covariates with significant $p$-values in the univariate model were included in the multivariate model. Statistical analyses were 
Table 1 Clinical trial protocols included in this analysis

\begin{tabular}{ll}
\hline NCT identifier & Trial title \\
\hline NCT01946204 A Multicenter, Randomized, Double-Blind, Placebo-Controlled, Phase III Study of \\
ARN-509 in Men With Non-Metastatic (M0) Castration-Resistant Prostate Cancer
\end{tabular}

NCT00744497 A Randomized Double-Blind Phase 3 Trial Comparing Docetaxel Combined With Dasatinib to Docetaxel Combined With Placebo in Castration-Resistant Prostate Cancer

NCT02057666 A Phase III, Randomised, Double-Blind, Placebo-Controlled Study Of Tasquinimod In Asian Chemo-Naiive Patients With Metastatic Castrate-Resistant Prostate Cancer

NCT01234311 A Phase 3 Randomized, Double-Blind, Placebo-Controlled Study of Tasquinimod in Men With Metastatic Castrate Resistant Prostate Cancer

\begin{tabular}{|c|c|}
\hline Phase & Reference \\
\hline $\begin{array}{l}\text { Phase } \\
\text { III }\end{array}$ & Apalutamide versus placebo \\
\hline $\begin{array}{l}\text { Phase } \\
\text { III }\end{array}$ & $\begin{array}{l}\text { Dasatinib, docetaxel, prednisone versus } \\
\text { placebo, docetaxel, prednisone }\end{array}$ \\
\hline $\begin{array}{l}\text { Phase } \\
\text { III }\end{array}$ & Tasquinimod versus placebo \\
\hline $\begin{array}{l}\text { Phase } \\
\text { III }\end{array}$ & Tasquinimod versus placebo \\
\hline $\begin{array}{l}\text { Phase } \\
\text { III }\end{array}$ & $\begin{array}{l}\text { Custirsen, docetaxel, prednisone versus } \\
\text { docetaxel, prednisone }\end{array}$ \\
\hline $\begin{array}{l}\text { Phase } \\
\text { II }\end{array}$ & $\begin{array}{l}\text { Radium-223 dichloride standard versus high } \\
\text { versus extended standard doses }\end{array}$ \\
\hline
\end{tabular}

NCT02023697 A Three Arm Randomized, Open-label Phase II Study of Radium-223 Dichloride $50 \mathrm{kBq} / \mathrm{kg}$ (55 kBq/kg After Implementation of NIST Update) Versus 80 kBq/kg (88 kBq/kg After Implementation of NIST Update), and Versus $50 \mathrm{kBq} / \mathrm{kg}$ $(55 \mathrm{kBq} / \mathrm{kg}$ After Implementation of NIST Update) in an Extended Dosing Schedule in Subjects With Castration-resistant Prostate Cancer Metastatic to the Bone

NCT01212991 Prevail: A Multinational Phase 3, Randomized, Double-blind, Placebo-controlled Efficacy And Safety Study Of Oral Mdv3100 In Chemotherapy-naïve Patients With ॥ Progressive Metastatic Prostate Cancer Who Have Failed Androgen Deprivation Therapy

NCT01685983 A Phase 2 Open Label Study of Abiraterone Acetate (JNJ-212082) and Prednisolone in Patients With Advanced Prostate Cancer Who Have Failed Androgen Deprivation and Docetaxel-Based Chemotherapy

NCT02003924 Prosper: A Multinational, Phase 3, Randomized, Double-blind, Placebo-controlled, Efficacy And Safety Study Of Enzalutamide In Patients With Nonmetastatic Castration-resistant Prostate Cancer

NCT01977651 A Multicenter, Single-Arm, Open-Label, Post-Marketing Safety Study to Evaluate the Risk of Seizure Among Subjects With Metastatic Castration-Resistant Prostate Cancer (mCRPC) Treated With Enzalutamide Who Are at Potential Increased Risk of Seizure

NCT02987543 A Phase III, Open Label, Randomized Study to Assess the Efficacy and Safety of Olaparib (Lynparza ${ }^{\mathrm{TM}}$ ) Versus Enzalutamide or Abiraterone Acetate in Men With Metastatic Castration-Resistant Prostate Cancer Who Have Failed Prior Treatment With a New Hormonal Agent and Have Homologous Recombination Repair Gene Mutations (PROfound)

NCT01188187 A Randomized Phase 3 Study Comparing Standard First-Line Docetaxel/Prednisone Phase to Docetaxel/Prednisone in Combination With Custirsen (OGX-011) in Men With Metastatic Castrate Resistant Prostate Cancer

NCT02200614 A Multinational, Randomised, Double-blind, Placebo-controlled, Phase III Efficacy and Safety Study of BAY1841788 (ODM-201) in Men With High-risk Non-metastatic Castration-resistant Prostate Cancer

NCT02257736 A Phase 3 Randomized, Placebo-controlled Double-blind Study of JNJ56021927 in Combination With Abiraterone Acetate and Prednisone Versus Abiraterone Acetate and Prednisone in Subjects With Chemotherapy-naive Metastatic Castration-resistant Prostate Cancer (mCRPC)

NCT00626548 A Phase III, Randomised, Placebo-controlled, Double-blind Study to Assess the Efficacy and Safety of Once-daily Orally Administered ZD4054 (Zibotentan) $10 \mathrm{mg}$ in Non-metastatic Hormone-resistant Prostate Cancer Patients

NCT00554229 A Phase III Trial to Test the Efficacy of ZD4054(Zibotentan), an Endothelin A Receptor Antagonist, Versus Placebo in Patients With Hormone Resistant Prostate Cancer (HRPC) and Bone Metastasis Who Are Pain Free and Mildly Symptomatic

NCT02677896 A Multinational, Phase 3, Randomized, Double-blind, Placebo-controlled Efficacy and Safety Study of Enzalutamide Plus Androgen Deprivation Therapy (ADT) Versus Placebo Plus ADT in Patients With Metastatic Hormone Sensitive Prostate Cancer (mHSPC)

Phase Enzalutamide versus placebo III

Phase Abiraterone versus prednisolone II

Phase Enzalutamide versus placebo III

Phase Enzalutamide

IV

Phase Olaparib versus enzalutamide or abiraterone III acetate
III

Phase III

Phase III

Phase III

Phase III

Phase Enzalutamide, androgen deprivation therapy III

Custirsen, docetaxel, prednisone versus docetaxel, prednisone

BAY1841788 (ODM-201) versus placebo

Apalutamide, abiraterone acetate, prednisone versus abiraterone acetate, prednisone

Zibotentan versus placebo

Zibotentan versus placebo versus placebo, androgen deprivation therapy 
Table 1 Clinical trial protocols included in this analysis (Continued)

\begin{tabular}{|c|c|c|c|}
\hline NCT identifier & Trial title & Phase & Reference \\
\hline NCT01217697 & $\begin{array}{l}\text { An Open Label Study of Abiraterone Acetate in Subjects With Metastatic } \\
\text { Castration-Resistant Prostate Cancer Who Have Progressed After Taxane-Based } \\
\text { Chemotherapy }\end{array}$ & EAP & Abiraterone acetate versus prednisone \\
\hline
\end{tabular}

performed using IBM SPSS software (version 23; IBM Corp., Armonk, NY, USA). All tests were two-tailed, with statistical significance set at a $p$-value of $<0.05$.

\section{Results}

\section{Baseline characteristics}

The baseline clinical and pathological features of the overall population and of the subgroups stratified by CT participation are presented in Table 2. Of the 299 patients, 65 (21.7\%) participated in CTs pertaining to systemic treatment target at CRPC while 234 (78.3\%) received pre-established, standard systemic treatment outside of a CT setting. A lower proportion of CT participants had ECOG PS $\geq 2$ at CRPC diagnosis than did non-participants $(4.6 \%$ vs. $14.9 \% ; p=0.033)$, while PSA levels at CRPC diagnosis were lower in CT participants compared to non-participants $(25.8 \mathrm{ng} / \mathrm{mL}$ vs. $88.4 \mathrm{ng} /$ $\mathrm{mL} ; p=0.005)$. Distributions of potential survival prognosticators of CRPC, namely, age, body mass index, TNM stages, Gleason score, metastatic sites, and CCI were comparable between the two groups.

The treatments administered for CRPC are described in Table 3. CT participants received significantly more cycles of docetaxel than did non-participants managed outside the CT setting. Because of Korea's National Health Insurance policy of providing reimbursement for enzalutamide used for post-chemotherapy patients with CRPC, enzalutamide was predominantly used in the post-docetaxel setting. There were no differences between the two groups in terms of the proportions of other systemic treatments used.

\section{Survival outcome according to clinical trial participation} Survival results as of September 2017 were used in this analysis and are presented in Table 4 and Fig. 1. During the median follow-up period of 16.0 months, the median CSS interval was 13.0 months. Overall, 187 (66.3\%) cancer-specific deaths were noted, which translated to a 2-year CSS rate of $46.8 \%$. CT participants exhibited significantly higher 2-year CSS rates than non-participants (61.3\% vs. $42.4 \% ; p=0.003)$.

\section{Predictors of cancer-specific survival}

Predictors of CSS are presented in Table 5. Univariate Cox-regression analyses demonstrated that patient age, PSA level at CRPC diagnosis, albumin and alkaline phosphatase levels, biopsy Gleason score $\geq 8$, ECOG PS $\geq 2$, less number of docetaxel cycles administered, and non-participation in CTs were associated with lower risk of CSS. Multivariate analysis revealed that PSA at CRPC diagnosis, alkaline phosphatase level, biopsy Gleason score $\geq 8$, ECOG PS $\geq 2$, less number of docetaxel cycles administered, and non-participation in CTs independently predicted lower risk of CSS.

\section{Discussion}

Systemic treatment for CRPC has rapidly evolved. Identifying patients for judicious application of optimal treatment strategies is imperative in the current era of multidisciplinary treatment options. However, the selection of agents is often limited by the availability of novel agents and reimbursement issues. In this regard, participation in CTs may provide a breakthrough opportunity for access to innovative therapeutic approaches in addition to third party payer coverage. Participation in CTs is based on the patient's notion that a survival benefit can be achieved. Our study demonstrated that participation in CTs pertaining to CRPC agents, compared with non-participation, may improve CSS regardless of metastatic status.

The biological mechanisms underlying improved CSS observed with CT participation in our study is unclear; however, several reasons have been proposed. First, an experimental treatment effect may have existed, in which CT participants received better treatment in early-phase CTs than they would have received with standard therapies [9]. This effect may potentially have affected our results in that systemic agents that have been identified to prolong survival-namely, abiraterone, enzalutamide, docetaxel, and radium-223 dichloride-were included in either the experimental or control arms in $66 \%$ of CTs included in our analysis. Furthermore, potential selection bias may arise from the 32 patients who were excluded from final analysis, if these patients had been allocated to receive novel agents without proven survival benefit. However, among the four excluded patients in the CT participation group, three patients had received abiraterone or docetaxel while one patient was blinded to arm allocation, precluding any alteration in our study results.

Second, a participation effect may have existed, in which aspects of CT participation other than exposure to investigational therapy may have improved outcomes [14]. Specifically, the participation effect comprises the following: 1) a protocol effect regarding the way the treatments are delivered; 2) a care effect including incidental aspects of care; 3) the Hawthorne effect, which is initiated by 
Table 2 Clinicopathologic characteristics of castration-resistant prostate cancer patients, stratified by clinical trial participation

\begin{tabular}{|c|c|c|c|c|}
\hline & \multirow{2}{*}{$\begin{array}{l}\text { Overall } \\
(\mathrm{n}=299)\end{array}$} & \multicolumn{3}{|l|}{ Clinical trial } \\
\hline & & $\begin{array}{l}\text { Participants } \\
(n=65)\end{array}$ & $\begin{array}{l}\text { Non-participants } \\
(n=234)\end{array}$ & $p$ \\
\hline Age & $66.5(61.0-71.8)$ & $65.0(62.0-71.0)$ & $67.0(61.0-72.0)$ & 0.384 \\
\hline Body mass index & $23.1(20.9-24.7)$ & $22.9(21.3-24.7)$ & $23.4(21.2-25.1)$ & 0.345 \\
\hline \multicolumn{5}{|l|}{ Laboratory values $^{a}$} \\
\hline PSA (ng/mL) & $69.2(15.0-182.0)$ & $25.8(9.6-73.6)$ & $88.4(18.0-247.3)$ & 0.005 \\
\hline Hemoglobin (g/dL) & $12.0(10.7-13.0)$ & $12.4(11.7-13.3)$ & $11.9(10.4-12.9)$ & 0.514 \\
\hline Albumin $(U / L)$ & $4.0(3.7-4.4)$ & $4.3(4.0-4.5)$ & $4.0(3.7-4.3)$ & 0.001 \\
\hline ALP (U/L) & $109.0(70.0-209.0)$ & $88.0(67.0-133.5)$ & $118(71.0-221.5)$ & 0.070 \\
\hline WBC count $\left(\times 10^{9} / \mathrm{L}\right)$ & $5.8(4.8-7.3)$ & $5.8(4.7-7.3)$ & $5.8(4.9-7.3)$ & 0.919 \\
\hline T stage & & & & 0.764 \\
\hline$\leq \mathrm{T} 2$ & $187(62.5 \%)$ & $42(64.7 \%)$ & $145(61.9 \%)$ & \\
\hline$\geq \mathrm{T3}$ & $112(37.5 \%)$ & $23(35.3 \%)$ & 89 (38.1\%) & \\
\hline N stage & & & & 0.491 \\
\hline No & $127(42.5 \%)$ & $30(46.2 \%)$ & 97 (41.5\%) & \\
\hline N1 & $172(57.5 \%)$ & 35 (53.8\%) & $137(58.5 \%)$ & \\
\hline M stage & & & & 1.000 \\
\hline MO & $73(24.4 \%)$ & $15(23.1 \%)$ & $58(24.8 \%)$ & \\
\hline M1 & $226(75.6 \%)$ & $50(76.9 \%)$ & $176(75.2 \%)$ & \\
\hline \multicolumn{5}{|l|}{ Metastatic site } \\
\hline Bone & $166(55.5 \%)$ & $37(57.0 \%)$ & $129(55.1 \%)$ & 0.856 \\
\hline Visceral & $8(2.7 \%)$ & $8(12.3 \%)$ & $0(0.0 \%)$ & 0.215 \\
\hline Lymph node & $142(47.5 \%)$ & $26(40.0 \%)$ & $116(49.6 \%)$ & 0.116 \\
\hline Gleason score & & & & 0.267 \\
\hline$\leq 7$ & $156(52.2 \%)$ & $30(46.2 \%)$ & $126(53.8 \%)$ & \\
\hline$\geq 8$ & $143(47.8 \%)$ & 35 (53.8\%) & $108(46.2 \%)$ & \\
\hline $\mathrm{CCl}$ & & & & 0.780 \\
\hline$\leq 1$ & $142(\%)$ & $32(49.2 \%)$ & $110(47.0 \%)$ & \\
\hline$\geq 2$ & $157(\%)$ & $33(50.8 \%)$ & $124(53.0 \%)$ & \\
\hline ECOG PS & & & & 0.033 \\
\hline$\leq 1$ & $261(87.3 \%)$ & $62(95.4 \%)$ & $199(85.1 \%)$ & \\
\hline$\geq 2$ & $38(12.7 \%)$ & $3(4.6 \%)$ & 35 (14.9\%) & \\
\hline \multicolumn{5}{|l|}{ Primary treatment ${ }^{b}$} \\
\hline Prostatectomy & $149(49.8 \%)$ & $47(72.3 \%)$ & $102(43.6 \%)$ & $<0.001$ \\
\hline Radiation therapy & $33(11.0 \%)$ & $10(15.4 \%)$ & $23(9.8 \%)$ & 0.261 \\
\hline
\end{tabular}

Data are presented as the median (interquartile range) or number (\%)

${ }^{\text {aAt }}$ diagnosis of castration-resistant prostate cancer

${ }^{b}$ Number of primary treatment does not sum to 299 patients due to the existence of men who did not receive any local treatment with curative intent Abbreviations: ALP alkaline phosphatase, CCI Charlson Comorbidity Index, ECOG PS Eastern Cooperative Oncology Group performance status, PSA prostate-specific antigen, WBC white blood cell

changes in physician or patient behavior in regard to the perception that they are under observation; and 4) the placebo effect, which mediates the psychological behavior of the participant based on the awareness that they are beneficiaries of therapeutic advances $[14,16]$.

Third, the improved survival outcomes observed with CT participation in our study may have resulted from inherent differences in baseline patient and tumor features. In our study, the performance status, as well as PSA and albumin levels of patients who participated in CTs were more favorable than those of non-participants; this might have affected the results. However, the proportions of potential survival confounders including age, tumor stage and grade, metastatic burden, and 
Table 3 Treatments administered for castration-resistant prostate cancer

\begin{tabular}{|c|c|c|c|c|}
\hline & \multirow{2}{*}{$\begin{array}{l}\text { Overall } \\
(n=299)\end{array}$} & \multicolumn{3}{|l|}{ Clinical trial } \\
\hline & & $\begin{array}{l}\text { Participants } \\
(\mathrm{n}=65)\end{array}$ & $\begin{array}{l}\text { Non-participants } \\
(n=234)\end{array}$ & $p$ \\
\hline \multicolumn{5}{|l|}{ Docetaxel } \\
\hline N & $\begin{array}{l}242 \\
(80.9 \%)\end{array}$ & 41 (63.1\%) & $201(85.9 \%)$ & 0.001 \\
\hline No. cycles & $\begin{array}{l}4.0 \\
(2.0-9.0)\end{array}$ & $\begin{array}{l}7.0 \\
(4.0-12.5)\end{array}$ & $4.0(2.0-9.0)$ & 0.003 \\
\hline \multicolumn{5}{|l|}{ ARAT agent use } \\
\hline Pre-chemotherapy & & & & 0.502 \\
\hline Abiraterone & $10(3.3 \%)$ & $2(3.1 \%)$ & $8(3.4 \%)$ & \\
\hline Enzalutamide & $15(5.0 \%)$ & $4(6.2 \%)$ & $11(4.7 \%)$ & \\
\hline Post-chemotherapy & & & & $<0.001$ \\
\hline Abiraterone & $23(7.7 \%)$ & $13(20.0 \%)$ & $10(4.3 \%)$ & \\
\hline Enzalutamide & $\begin{array}{l}108 \\
(36.1 \%)\end{array}$ & $9(13.8 \%)$ & 99 (42.3\%) & \\
\hline Cabazitaxel & $1(0.3 \%)$ & $0(0.0 \%)$ & $1(0.4 \%)$ & 1.000 \\
\hline Radium-223 & $5(1.7 \%)$ & $3(4.6 \%)$ & $2(0.9 \%)$ & 0.070 \\
\hline
\end{tabular}

Abbreviations: ARAT androgen receptor axis-targeted

comorbidities were comparable between the groups. To overcome the challenge in separating possible true effects from false effects of the discrepancy in baseline patient and tumor features, statistical adjustments were made for a comprehensive set of confounders of survival among patients with CRPC, to confirm that CT participation itself was an independent prognosticator. Another selection bias arises from the effect of protocol eligibility criteria [12]. Most CTs included in our study strictly prohibited enrollment of patients with advanced disease, such as those with brain metastasis, an adverse prognostic factor for several cancers [18-20]. However, the two study groups had comparable proportions of metastatic location and burden, corroborating our hypothesis.

Fourth, a bias in data collection with regard to survival may have affected outcomes; survival follow-up could be more completely censored in CT participants than in non-participants. Moreover, patients in the advanced stages of the disease who participated in CTs could have been inherently more adherent to treatment follow-up schedules, whereas non-participants might opted for supportive care even if anti-cancer treatment may have prolonged survival $[16,21]$.

The present study revealed that CT participants received more docetaxel cycles than non- participants. Docetaxel remains the standard treatment for metastatic CRPC and has been the mainstay for CTs of sequential strategies since its approval in 2004 [22]. The improved survival in CT participants may be attributed to better chemotherapy efficacy and subsequent prolonged duration of docetaxel administration, as shown in multivariate analysis. Our study also demonstrated that Gleason score, and PSA and alkaline phosphatase levels at CRPC diagnosis are independent predictors of CSS. These findings compare favorably to those of previous retrospective studies that investigated prognosticators for survival in patients with metastatic CRPC, which implies that our cohort ably represented the whole population of the disease status and that our conclusions are generalizable [23, 24].

With our use of retrospective data, it is difficult to determine which of the abovementioned effects contributed to the survival benefit associated with CT participation. Indeed, a randomized controlled trial in which patients are randomized to be offered CT participation would be warranted to ensure baseline comparability and to investigate potential confounders. However, if at least one of the abovementioned effects may have truly affected improved CSS outcome in our CT participants, it would provide evidence to offer CT participation whenever indicated to patients with CRPC for its inherent survival advantage.

The strengths of the current study include the incorporation of comprehensive survival prognosticators of CRPC, including patient and tumor characteristics, comorbidities, performance status, laboratory values, and treatment information that were available for all patients. Furthermore, CT participants included in our study received novel hormonal, chemotherapeutic, and radiopharmaceutical therapeutic agents approved in the last 8 years, which suggests that our results are applicable in this contemporary era of multidisciplinary treatment strategies. At the same time, several limitations are worth mentioning. First, selection bias may have existed

Table 4 Survival outcomes of patients with castration-resistant prostate cancer, stratified by clinical trial participation

\begin{tabular}{|c|c|c|c|c|}
\hline & \multirow{2}{*}{$\begin{array}{l}\text { Overall } \\
(n=299)\end{array}$} & \multicolumn{3}{|l|}{ Clinical trial } \\
\hline & & $\begin{array}{l}\text { Participants } \\
(n=65)\end{array}$ & $\begin{array}{l}\text { Non-participants } \\
(n=234)\end{array}$ & $p$ \\
\hline No. cancer-specific deaths & $187(62.5 \%)$ & $44(67.7 \%)$ & $143(61.1 \%)$ & 0.364 \\
\hline 2-year cancer-specific survival & $46.8 \%$ & $61.3 \%$ & $42.4 \%$ & 0.003 \\
\hline CRPC to death (months) & $13.0(6.0-24.3)$ & $23.5(13.3-30.5)$ & $11.0(5.0-19.3)$ & $<0.001$ \\
\hline Total follow-up (months) & $16.0(7.2-26.0)$ & $26.0(16.0-39.8)$ & $13.5(6.0-24.0)$ & $<0.001$ \\
\hline
\end{tabular}

Data are presented as number (\%) or median (interquartile range)

Abbreviations: CRPC castration-resistant prostate cancer 


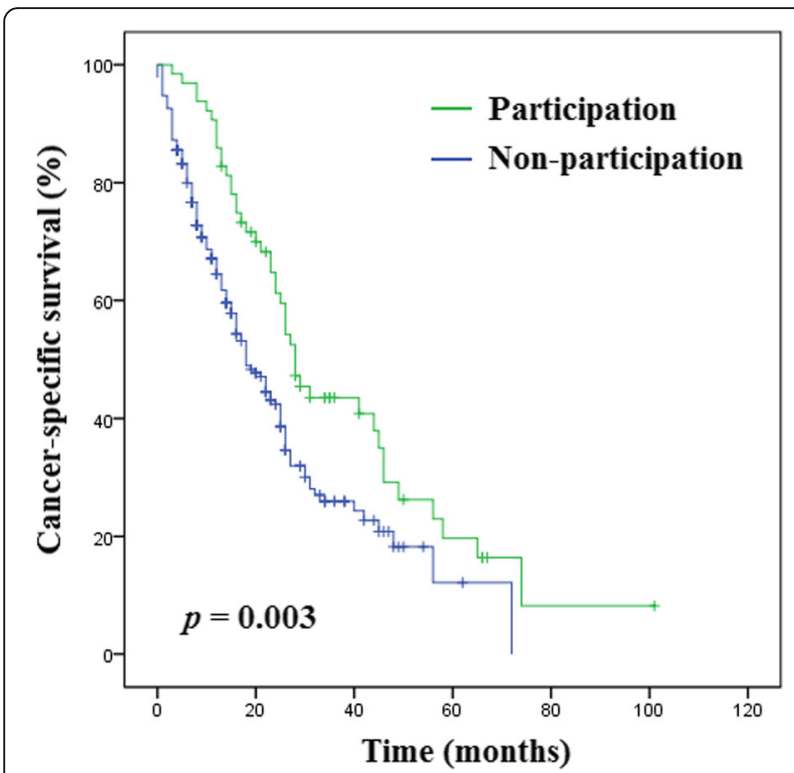

Fig. 1 Cancer-specific survival of patients with castration-resistant prostate cancer, stratified by clinical trial participation versus nonparticipation due to the retrospective nature of the study. This study was a non-randomized study; therefore, there was a lack of a standard therapeutic approach in which physician and patient preferences existed regarding the implementation of a CT. Moreover, a discrepancy existed in treatment protocols used in various CTs, such as the frequency of imaging and laboratory testing, and between each physician who treated patients with standard care. Second, the existence of unaccounted imbalances in baseline patient and tumor characteristics cannot be overlooked. However, these potential baseline discrepancies which may have affected our outcomes were accounted for, and our results were derived from multivariate Cox-regression analyses. Lastly, we did not account for the data of patients who participated in CTs but later declined to continue and opted for best supportive care, which may have affected survival outcomes. The abstract of this article was presented at the 33rd Annual EAU Congress [25].

\section{Conclusions}

This observational study provides novel findings that the CSS outcomes of patients diagnosed with CRPC who

Table 5 Predictors of cancer-specific mortality in patients with castration-resistant prostate cancer

\begin{tabular}{|c|c|c|c|c|c|c|}
\hline & \multicolumn{3}{|c|}{ Univariate } & \multicolumn{3}{|c|}{ Multivariate } \\
\hline & $\overline{\mathrm{HR}}$ & $(95 \% \mathrm{Cl})$ & $P$ & $\overline{\mathrm{HR}}$ & $(95 \% \mathrm{Cl})$ & $p$ \\
\hline Age & 1.038 & $(1.016-1.061)$ & 0.001 & 1.020 & $(0.998-1.042)$ & 0.069 \\
\hline Body mass index & 0.968 & $(0.901-1.041)$ & 0.382 & & & \\
\hline$P S A^{a}$ & 1.001 & $(1.000-1.001)$ & $<0.001$ & 1.001 & $(1.000-1.001)$ & 0.018 \\
\hline Hemoglobin ${ }^{a}$ & 1.001 & $(0.999-1.002)$ & 0.306 & & & \\
\hline Albumin ${ }^{a}$ & 0.408 & $(0.301-0.553)$ & $<0.001$ & & & \\
\hline Alkaline phosphatase $e^{a}$ & 1.001 & $(1.000-1.001)$ & $<0.001$ & 1.001 & $(1.001-1.002)$ & $<0.001$ \\
\hline $\mathrm{T}$ stage ( $\geq \mathrm{T} 3$ vs. $\leq \mathrm{T} 2$ ) & 0.865 & $(0.524-1.430)$ & 0.271 & & & \\
\hline N stage (1 vs. 0 ) & 1.251 & $(0.922-1.697)$ & 0.152 & & & \\
\hline M stage (1 vs. 0 ) & 1.528 & $(0.983-2.376)$ & 0.062 & & & \\
\hline Gleason score ( $\geq 8$ vs. $\leq 7$ ) & 1.957 & $(1.441-2.658)$ & $<0.001$ & 2.004 & $(1.452-2.767)$ & $<0.001$ \\
\hline $\mathrm{CCl} \geq 4$ & 1.197 & $(0.811-1.765)$ & 0.365 & & & \\
\hline$E C O G \geq 2$ & 1.802 & $(1.216-2.670)$ & 0.003 & 1.304 & $(1.164-2.158)$ & 0.035 \\
\hline Docetaxel cycles & 0.926 & $(0.900-0.953)$ & 0.026 & 0.943 & $(0.915-0.972)$ & 0.011 \\
\hline \multicolumn{7}{|l|}{ Primary treatment } \\
\hline Prostatectomy & 1 & (reference) & & & & \\
\hline Radiation therapy & 0.778 & $(0.580-1.141)$ & 0.584 & & & \\
\hline \multicolumn{7}{|l|}{ ARAT agent sequencing } \\
\hline Pre-chemotherapy & 1 & (reference) & & & & \\
\hline Post-chemotherapy & 0.865 & $(0.524-1.430)$ & 0.572 & & & \\
\hline Radium-223 administration & 0.803 & $(0.255-2.527)$ & 0.707 & & & \\
\hline Clinical trial participation & 0.593 & $(0.417-0.843)$ & 0.004 & 0.585 & $(0.429-0.797)$ & 0.038 \\
\hline
\end{tabular}

ababoratory values at diagnosis of castration-resistant prostate cancer

Abbreviations: ARAT androgen receptor axis-targeted, Cl confidence interval, CCI Charlson Comorbidity Index, ECOG PS Eastern Cooperative Oncology Group performance status, $H R$ hazard ratio, PSA prostate-specific antigen 
participated in CTs were better than those of nonparticipants who received pre-established, standard systemic therapy outside of a CT setting. Our findings imply that CT participation is associated with CSS, and that CT participation should be offered to patients with CRPC whenever indicated.

\section{Abbreviations \\ CCl: Charlson Comorbidity Index; CRPC: Castration-resistant prostate cancer CSS: Cancer-specific survival; CT: Clinical trial; ECOG PS: Eastern Cooperative Oncology Group performance score; PSA: Prostate-specific antigen}

\section{Funding}

This study was supported by the Young Researcher Program Grant from the National Research Foundation of Korea (NRF-2017R1C1B5017516). The funding body had no active role in any stage of the study, including: design, data collection, analysis, interpretation of data, and writing the manuscript.

\section{Availability of data and materials}

The dataset analyzed during the current study is available from the corresponding author on reasonable request.

\section{Author's contributions}

Study concept and initial design: KCK, JSL, KHR, SJH, BHC; Acquisition of data: KCK, JWK, YSH, DKK; Data analysis: KSH, KSL, DKK, YSH; Manuscript writing: KCK, JSL; Critical revision for important intellectual content: JWK, KSH, KSL, DKK, YSH, KHR, SJH, BHC. All authors have participated sufficiently in the work to take public responsibility for appropriate portions of the content, and have given final approval of the version to be published. All authors agreed to be accountable for all aspects of the work in ensuring that questions related to the accuracy or integrity of any part of the work are appropriately investigated and resolved.

\section{Ethics approval and consent to participate}

This study was approved by the Yonsei University Health System Institutiona Review Board (2017-0186-001). Informed consent was waived from the Yonsel University Health System Institutional Review Board since patients' information was collected during the routine clinical practice and patients were identified by anonymized investigator-generated code not linkable to their personal data. The same Institutional Review Board granted access to the institutional databases used in this study.

\section{Competing interests}

The authors declare that they have no competing interests.

\section{Publisher's Note}

Springer Nature remains neutral with regard to jurisdictional claims in published maps and institutional affiliations.

\section{Author details}

'Department of Urology, Gangnam Severance Hospital, Yonsei University College of Medicine, 211 Eonju-ro, Gangnam-gu, Seoul 135-720, Republic of Korea. ${ }^{2}$ Department of Urology, Severance Hospital, Yonsei University College of Medicine, Seoul, Republic of Korea.

Received: 15 January 2018 Accepted: 17 April 2018

Published online: 26 April 2018

References

1. Kim CS, Theeuwes A, Kwon DD, Choi YD, Chung BH, Lee HM, et al. The PREVAIL trial of enzalutamide in men with chemotherapy-naïve, metastatic castration-resistant prostate cancer: Post hoc analysis of Korean patients. Investig Clin Urol. 2016;57:174-83.

2. de Bono JS, Logothetis CJ, Molina A, Fizazi K, North S, Chu L, et al Abiraterone and increased survival in metastatic prostate cancer. N Engl J Med. 2011:364:1995-2005.

3. Ryan CJ, Smith MR, de Bono JS, Molina A, Logothetis CJ, de Souza P, et al. Abiraterone in metastatic prostate cancer without previous chemotherapy. N Engl J Med. 2013;368:138-48.
4. Beer TM, Armstrong AJ, Rathkopf DE, Loriot Y, Sternberg CN, Higano CS, et al. Enzalutamide in metastatic prostate cancer before chemotherapy. $\mathrm{N}$ Engl J Med. 2014;371:424-33.

5. Scher HI, Fizazi K, Saad F, Taplin ME, Sternberg CN, Miller K, et al. Increased survival with enzalutamide in prostate cancer after chemotherapy. N Engl J Med. 2012;367:1187-97.

6. Antonarakis ES, Armstrong AJ. Emerging therapeutic approaches in the management of metastatic castration-resistant prostate cancer. Prostate Cancer Prostatic Dis. 2011:14:206-18.

7. Hanahan D, Weinberg RA. Hallmarks of cancer: the next generation. Cell. 2011:144:646-74.

8. Joniau S, Abrahamsson PA, Bellmunt J, Figdor C, Hamdy F, Verhagen P, et al. Current vaccination strategies for prostate cancer. Eur Urol. 2012;61: 290-306.

9. Goyal J, Nuhn P, Huang P, Tyagi P, Oh D, Carducci MA, et al. The effect of clinical trial participation versus non-participation on overall survival in men receiving first-line docetaxel-containing chemotherapy for metastatic castration-resistant prostate cancer. BJU Int. 2012;110:E575-82.

10. Stiller CA. Centralised treatment, entry to trials and survival. $\mathrm{Br} J$ Cancer. 1994:70:352-62

11. Go RS, Frisby KA, Lee JA, Mathiason MA, Meyer CM, Ostern JL, et al. Clinical trial accrual among new cancer patients at a community-based cancer center. Cancer. 2006;106:426-33.

12. Jenkins $V$, Fallowfield $L$. Reasons for accepting or declining to participate in randomized clinical trials for cancer therapy. Br J Cancer. 2000;82:1783-8.

13. Tanai C, Nakajima TE, Nagashima K, Kato K, Hamaguchi T, Yamada Y, et al. Characteristics and outcomes of patients with advanced gastric cancer who declined to participate in a randomized clinical chemotherapy trial. J Oncol Pract. 2011;7:148-53.

14. Peppercorn JM, Weeks JC, Cook EF, Joffe S. Comparison of outcomes in cancer patients treated within and outside clinical trials: conceptual framework and structured review. Lancet. 2004;363:263-70.

15. West J, Wright J, Tuffnell D, Jankowicz D, West R. Do clinical trials improve quality of care? A comparison of clinical processes and outcomes in patients in a clinical trial and similar patients outside a trial where both groups are managed according to a strict protocol. Qual Saf Health Care. 2005;14:175-8.

16. Braunholtz DA, Edwards SJ, Lilford RJ. Are randomized clinical trials good for us (in the short term)? Evidence for a "trial effect". J Clin Epidemiol. 2001:54:217-24.

17. Scher HI, Halabi S, Tannock I, Morris M, Sternberg CN, Carducci MA, et al. Design and end points of clinical trials for patients with progressive prostate cancer and castrate levels of testosterone: recommendations of the prostate Cancer clinical trials working group. J Clin Oncol. 2008;26:1148-59.

18. Koo KC, Lee KS, Cho KS, Rha KH, Hong SJ, Chung BH. Comprehensive analysis and validation of contemporary survival prognosticators in Korean patients with metastatic renal cell carcinoma treated with targeted therapy: prognostic impact of pretreatment neutrophil-to-lymphocyte ratio. Int Urol Nephrol. 2016:48:985-92.

19. Bates JE, Youn P, Usuki KY, Walter KA, Huggins CF, Okunieff $P$, et al. Brain metastasis from melanoma: the prognostic value of varying sites of extracranial disease. J Neuro-Oncol. 2015;125:411-8.

20. Divine LM, Kizer NT, Hagemann AR, Pittman ME, Chen L, Powell MA, et al. Clinicopathologic characteristics and survival of patients with gynecologic malignancies metastatic to the brain. Gynecol Oncol. 2016;142:76-82.

21. Leventhal $H$, Nerenz DR, Leventhal EA, Love RR, Bendena LM. The behavioral dynamics of clinical trials. Prev Med. 1991;20:132-46.

22. Tannock IF, de Wit R, Berry WR, Horti J, Pluzanska A, Chi KN, et al. Docetaxel plus prednisone or mitoxantrone plus prednisone for advanced prostate cancer. N Engl J Med. 2004:351:1502-12.

23. Halabi S, Small EJ, Kantoff PW, Kattan MW, Kaplan EB, Dawson NA, et al. Prognostic model for predicting survival in men with hormone-refractory metastatic prostate cancer. J Clin Oncol. 2003;21:1232-7.

24. Armstrong AJ, Garrett-Mayer E, de Wit R, Tannock I, Eisenberger M. Prediction of survival following first-line chemotherapy in men with castration-resistant metastatic prostate cancer. Clin Cancer Res. 2010;16:203-11.

25. Koo KC, Lee JS, Kim JW, Kang SK, Lee KS, Kim DK, et al. Impact of clinica trial participation on survival in patients with castration-resistant prostate cancer: a multi-center analysis. Eur Urol Suppl. 2018:17:e1081-82. https://doi. org/10.1016/\$1569-9056(18)31586-0. 